\title{
Hydrodynamic modeling and motion limits of AUV MARES
}

\author{
Bruno Ferreira, Miguel Pinto, Aníbal Matos, Nuno Cruz \\ FEUP - DEEC \\ Rua Dr. Roberto Frias, s/n 4200-465 Porto PORTUGAL \\ ee04018@fe.up.pt, ee04134@fe.up.pt, anibal@fe.up.pt, nacruz@fe.up.pt
}

\begin{abstract}
This papers addresses the dynamic characterization of the autonomous underwater vehicle MARES. The paper presents the main dynamic properties of this underwater robotic platform as well as the procedures employed to obtain the parameters that define the vehicle model. Furthermore, the paper also presents a detailed characterization of the elementary motions that this vehicle is able to perform.
\end{abstract}

\section{INTRODUCTION}

The use of autonomous underwater vehicles (AUVs) in different application fields, such as military, homeland defense, underwater surveys, environment monitoring, and oceanographic studies, is becoming more and more common. Nonetheless, there is still a great research effort related to the development of this technology, with a large number of different vehicles being developed either for generic or more specific applications. Regarding small size AUVs, the traditional torpedo shapes with a main propeller and control surfaces are being replaced by vehicles with higher maneuverability that can be advantageously used in more demanding applications.

This paper addresses the dynamic modeling and motion characterization of the MARES AUV, a small size vehicle developed at Porto University and already demonstrated at sea operations in 2007. We determine and present a model that considers all major characteristics without habitual simplifications of the body form. The major concepts and expressions of the modeling process of an AUV actuated by thrusters are compiled and may be consulted in this document.

The paper starts with a brief description of the MARES AUV and the basic design options. Next, we present the dynamic model that was used to characterize its behavior. Then, in section IV, we present the derivation of the parameters of the model. Section V addresses the characterization of the vehicle behavior in some maneuvers.

\section{MARES}

MARES, or Modular Autonomous Robot for Environment Sampling (fig. 1), is a $1.5 \mathrm{~m}$ long AUV, designed and built by the Ocean Systems Group [1-2]. The vehicle can be programmed to follow predefined trajectories, while collecting relevant data with the onboard sensors. MARES can dive up to $100 \mathrm{~m}$ deep, and unlike similar-sized systems, has vertical thrusters to allow for purely vertical motion in the water column. Forward velocity can be independently defined, from 0 to about $1.5 \mathrm{~m} / \mathrm{s}$. Major application areas

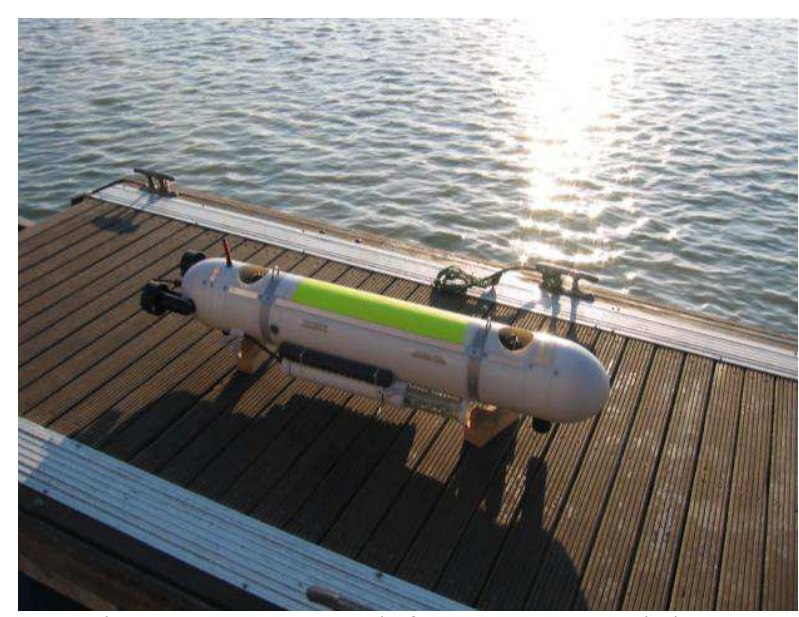

Fig. 1: MARES AUV ready for an autonomous mission.

include pollution monitoring, scientific data collection, sonar mapping, underwater video or mine countermeasures.

MARES configuration can change significantly according to the application scenario, so that it is difficult to define what is a standard configuration. In table 1 we summarize the main characteristics of the AUV version that was demonstrated at sea in November 2007.

TABLE I

MARES CHARACTERISTICS

\begin{tabular}{|l|l|}
\hline Length & $1.5 \mathrm{~m}$ \\
\hline Diameter & $20 \mathrm{~cm}$ \\
\hline Weight in air & $32 \mathrm{~kg}$ \\
\hline Depth rating & $100 \mathrm{~m}$ \\
\hline Propulsion & 2 horizontal +2 vertical thrusters \\
\hline Horizontal velocity & $0-1.5 \mathrm{~m} / \mathrm{s}$, variable \\
\hline Energy & Li-Ion batteries, $600 \mathrm{Wh}$ \\
\hline Autonomy/Range & about 10 hrs $/ 40 \mathrm{~km}$ \\
\hline
\end{tabular}

The vehicle hull is based on a central watertight polyacetal cylinder, where all electronic boards are installed, with the battery packs located at the bottom to lower the center of mass. To simplify the design, this is the only watertight enclosure and therefore all other equipment has to be waterproof. The other polyacetal sections are designed to carry wet sensors and thrusters and they are fully interchangeable. This allows for very easy sensor swapping and/or repositioning, or even to test different configurations of thrusters.

The power required by an AUV is mainly spent in propulsion, with only a small amount permanently needed for onboard electronics. In MARES, all energy is stored in rechargeable Li-Ion battery packs, currently with a total amount of $600 \mathrm{Wh}$, at $14.4 \mathrm{~V}$. Battery power is directly 
available to the motor controllers and, through a set of voltage converters, to the rest of the onboard electronics.

\section{DYNAMIC MODEL}

In this paper we intend to determine a model of the MARES AUV considering all its major characteristics. In particular, in this section, we present the general equation of the motion dynamic.

The derivation of the dynamic model for the AUV follows the standard approach presented in [3] where two reference frames are considered: an earth fixed frame assumed to have inertial properties and a body frame that moves together with the vehicle. To relate linear and angular velocities as well as forces and torques defined in the two frames it is necessary to define

$$
\begin{aligned}
\eta_{1} & =\left[\begin{array}{l}
x \\
y \\
Z
\end{array}\right] ; & \eta_{2} & =\left[\begin{array}{c}
\phi \\
\theta \\
\psi
\end{array}\right] ; \\
v_{1} & =\left[\begin{array}{c}
u \\
v \\
W
\end{array}\right] ; & v_{2} & =\left[\begin{array}{c}
p \\
q \\
r
\end{array}\right] ; \\
\tau_{1} & =\left[\begin{array}{l}
X \\
Y \\
Z
\end{array}\right] ; & \tau_{2} & =\left[\begin{array}{c}
K \\
M \\
N
\end{array}\right] .
\end{aligned}
$$

where $\eta_{1}$ and $\eta_{2}$ are the relative position and orientation of the body fixed frame with respect to the inertial frame; $v_{1}$ and $v_{2}$ are the linear and angular velocities expressed in body fixed coordinates; $\tau_{1}$ and $\tau_{2}$ are the forces and torques acting on the vehicle, also expressed in body fixed coordinates. Defining $\eta=\left[\begin{array}{ll}\eta_{1}^{T} & \eta_{2}^{T}\end{array}\right]^{T}, v=\left[\begin{array}{ll}v_{1}^{T} & v_{2}^{T}\end{array}\right]^{T}$ and $J=\operatorname{diag}\left(J_{1}, J_{2}\right)$ where

$$
\begin{gathered}
J_{1}=\left[\begin{array}{ccc}
\mathrm{c} \psi \mathrm{c} \theta & -\mathrm{s} \psi \mathrm{c} \phi+\mathrm{c} \psi \mathrm{s} \theta \mathrm{s} \phi & \mathrm{s} \psi \mathrm{s} \phi+\mathrm{c} \psi \mathrm{s} \theta \mathrm{c} \phi \\
\mathrm{s} \psi \mathrm{c} \theta & \mathrm{c} \psi \mathrm{c} \phi+\mathrm{s} \psi \mathrm{s} \theta \mathrm{s} \phi & -\mathrm{c} \psi \mathrm{s} \phi+\mathrm{s} \psi \mathrm{s} \theta \mathrm{c} \phi \\
-\mathrm{s} \theta & \mathrm{c} \theta \mathrm{s} \phi & \mathrm{c} \theta \mathrm{c} \phi
\end{array}\right] \\
J_{2}=\left[\begin{array}{ccc}
1 & \mathrm{~s} \phi \mathrm{t} \theta & \mathrm{c} \phi \mathrm{t} \theta \\
0 & \mathrm{c} \phi & -\mathrm{s} \phi \\
0 & \mathrm{~s} \phi / \mathrm{c} \theta & \mathrm{c} \phi / \mathrm{c} \theta
\end{array}\right]
\end{gathered}
$$

The relationships between linear and angular velocities in the two frames are

$$
\dot{\eta}=J\left(\eta_{2}\right) v
$$

The dynamics of the vehicle are easily expressed in the body fixed frame by

$$
\tau_{\text {ext }}=M_{R B} \ddot{\eta}+C_{R B}(\dot{\eta}) \dot{\eta},
$$

where $\tau_{\text {ext }}$ is the vector composed by the external forces and torques acting on the vehicle, expressed in body frame coordinates, $M_{R B}$ is the inertial matrix and $C_{R B}(\dot{\eta})$ is the Coriolis and centripetal matrix (cf. [3] for details). The external forces and moments can be decomposed as the sum of the added mass, potential damping, drag, restoring, and propulsion, respectively,

$$
\tau_{\text {ext }}=\tau_{A}+\tau_{B}+\tau_{V}+\tau_{C}+\tau_{\text {prop }} .
$$

From (3), (4) and (5), we can conclude that $\tau_{\text {ext }} \in \mathbb{R}^{6}$. The first three lines regard to the components of forces, while the last three regard to the components of torques, both in the body-fixed coordinate system that we will define in the next section.

\section{COEFFICIENTS DETERMINATION}

In the followings sections and subsections, we will consider that the fixed body coordinate system $x_{B} y_{B} z_{B}$ coincides with the vehicle center of gravity (CG), as stated in fig. 2 and fig. 3 .

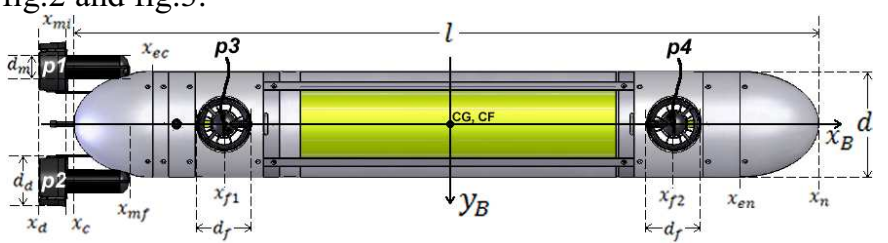

Fig. 2: Vertical projection of MARES

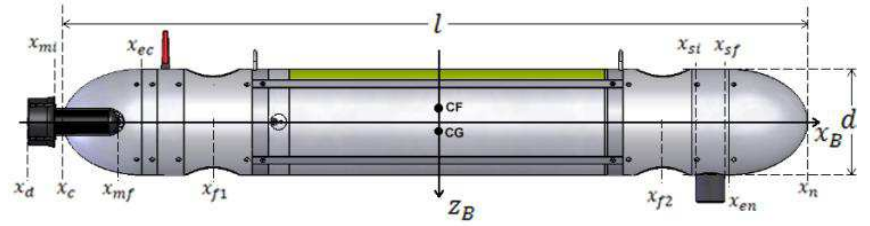

Fig. 3: Lateral projection of MARES

The determination of the vehicle model coefficients is based on theoretical analysis of its shape. Here, we only present the derivation of some added mass and drag coefficients. More details are presented in [4].

The antenna and the handles effects on AUV superior part will be neglected.

A. Added mass

The axial term $X_{\dot{u}}$ is determined using the equation (6) from [1 p.41]. We need to approximate the vehicle by an ellipsoidal with a minor axis $a=l / 2$ and a major axis $b=d / 2$.

$$
X_{\dot{u}}=-\frac{\alpha_{0}}{2-\alpha_{0}} m,
$$

where $m$ is the total vehicle mass, and $\alpha_{0}$ is given by [3]

$$
\alpha_{0}=\frac{2\left(1-e^{2}\right)}{e^{3}}\left[\frac{1}{2} \ln \left(\frac{1+e}{1-e}\right)-e\right]
$$

and

$$
e=1-\left(\frac{b}{a}\right)^{2}
$$

Based on [5] and [6], we assume that the major contributions to the added mass are the vehicle hull, the horizontal propellers and the sonar transducer. The fluid masses displaced by the AUV, per unit of a transversal "slice" of these three contributions are respectively:

$$
\begin{aligned}
& m_{a}\left(x_{B}\right)=\rho_{f} \int_{0}^{2 \pi} \int_{0}^{R\left(x_{B}\right)} r d r d \varphi=\rho_{f} \pi R\left(x_{B}\right)^{2}, \\
& m_{a P}\left(x_{B}\right)=\rho_{f} \int_{0}^{2 \pi} \int_{0}^{R_{P}\left(x_{B}\right)} r d r d \varphi=\rho_{f} \pi R_{P}\left(x_{B}\right)^{2}, \\
& m_{a S}\left(x_{B}\right)=2 \rho_{f} \int_{0}^{x_{B}} H_{S} \sqrt{y_{S}{ }^{2}-\left(x-x_{c S}\right)^{2}} d x .
\end{aligned}
$$


where $R\left(x_{B}\right)$ is the hull radius, $R_{P}\left(x_{B}\right)$ is the propeller radius, $H_{S}$ is the sonar hull height and $x_{C S}$ the sonar hull base center.

The motions in $y_{B}$ e $z_{B}$ directions cause different forces and moments, due to the existence of verticals holes in the hull. In $y_{B}$ or yaw motion, the fluid present in the holes is transported with the vehicle, but in $z_{B}$ or pitch motion, the fluid does not suffer any acceleration. This last case implies that the fluid passes through the holes does not exercise any force or moment on the vehicle.

We approach the holes by cylinders with a diameter $d_{f}$ and constant height $h_{f}$. We can now define the added mass per unit length, due to holes:

$$
m_{a f}\left(x_{B}\right)= \begin{cases}\rho_{f} h_{f} \sqrt{\left(\frac{d_{f}}{2}\right)^{2}-\left(x_{B}-x_{f 1}\right)^{2}}, & x_{f 1}-\frac{d_{f}}{2} \leq x_{B} \\ \rho_{f} h_{f} \sqrt{\left(\frac{d_{f}}{2}\right)^{2}-\left(x_{B}-x_{f 2}\right)^{2}}, & <x_{f 1}+\frac{d_{f}}{2} \\ & x_{f 2}-\frac{d_{f}}{2} \leq x_{B} \\ 0, & <x_{f 2}+\frac{d_{f}}{2} \\ \text { Other. }\end{cases}
$$

In (13), we present the expressions to determine some added mass terms. The other terms can be determined by analogy.

$$
\begin{gathered}
Z_{\dot{w}}=-\left[\int_{x_{C}}^{x_{n}} m_{a}\left(x_{B}\right) d x_{B}+2 \int_{x_{d}}^{x_{m f}} m_{a P}\left(x_{B}\right) d x_{B}\right. \\
\left.+\int_{x_{S i}}^{x_{S f}} m_{a S}\left(x_{B}\right) d x_{B}-\int_{x_{C}}^{x_{n}} m_{a f}\left(x_{B}\right) d x_{B}\right], \\
N_{\dot{r}}=-\left[\int_{x_{C}}^{x_{n}} x_{B}^{2} m_{a}\left(x_{B}\right) d x_{B}+2 \int_{x_{d}}^{x_{m f}} x_{B}{ }^{2} m_{a P}\left(x_{B}\right) d x_{B}\right. \\
\left.+\int_{x_{S i}}^{x_{S f}} x_{B}{ }^{2} m_{a S}\left(x_{B}\right) d x_{B}\right], \\
Y_{\dot{r}}=\left[\int_{x_{C}}^{x_{n}} x_{B} m_{a}\left(x_{B}\right) d x_{B}+2 \int_{x_{d}}^{x_{m f}} x_{B} m_{a P}\left(x_{B}\right) d x_{B}\right. \\
\left.+\int_{x_{S i}}^{x_{S f}} x_{B} m_{a S}\left(x_{B}\right) d x_{B}\right],
\end{gathered}
$$

for the limits of integration, see fig.2 and fig.3.

The most important added mass coefficients are shown in table II.

TABLE II

ADDED MASS COEFFICIENT VALUES

\begin{tabular}{|c|c|}
\hline Coefficient & Value \\
\hline$X_{\dot{u}}$ & $-1.74 \cdot 10^{0}$ \\
\hline$Y_{\dot{v}}$ & $-4.28 \cdot 10^{1}$ \\
\hline$Z_{\dot{w}}$ & $-3.88 \cdot 10^{1}$ \\
\hline$K_{\dot{p}}$ & $-2.05 \cdot 10^{-1}$ \\
\hline$M_{\dot{q}}$ & $-5.59 \cdot 10^{0}$ \\
\hline
\end{tabular}$\quad$\begin{tabular}{c|c|}
\hline Coefficient & Value \\
\hline$N_{\dot{r}}$ & $-6.32 \cdot 10^{0}$ \\
\hline$Y_{\dot{r}}$ & $2.89 \cdot 10^{-2}$ \\
\hline$Z_{\dot{q}}$ & $-2.10 \cdot 10^{-1}$ \\
\hline$M_{\dot{w}}$ & $-2.10 \cdot 10^{-1}$ \\
\hline$N_{\dot{v}}$ & $2.89 \cdot 10^{-2}$ \\
\hline
\end{tabular}

\section{B. Drag}

In this work, we consider only quadratic drag terms. We assume that the linear and angular speeds are sufficiently high to neglect linear terms. The terms greater than second order will be neglect too, assuming that their effects are small comparing to quadratic terms.

From [3] and [7] we have the axial force in the $x_{B}$ direction expressed as:

$$
f_{d u}\left(u_{r}\right)=-\frac{1}{2} \rho_{f} C_{D e} A_{y z} u_{r}\left|u_{r}\right|,
$$

where $\rho_{f}=1.026 \cdot 10^{3} \mathrm{~kg} / \mathrm{m}^{3}$ is the fluid density, $C_{D e}$ is the drag coefficient of an ellipsoidal body depending on the hull form and $A_{y z}$ is the vehicle projected area in the plane formed by $y_{B}$ and $z_{B}$ axes.

The determination of drag coefficients can result on wrong estimations [5] because the theory that is behind is often based on empirical expressions or experimental results. The best way to estimate them is by experimental tests. However, those estimates are useful as a first approach to the characterization of the vehicle motion.

The drag coefficient $C_{D e}$ depends on Reynolds Number $R_{e}$, given by:

$$
R_{e}=\frac{U l}{v},
$$

where $U$ is the axial speed in the $x_{B}$-axis, $l$ the vehicle length and $v$ the fluid viscosity. We assume $U=1 \mathrm{~m} / \mathrm{s}$ which is a typical value for the vehicle velocity, $l=1.5 \mathrm{~m}$ and $v=$ $1.005 \cdot 10^{-6} \mathrm{~m}^{2} / \mathrm{s}$ at $T=20^{\circ} \mathrm{C}$. These values give $R_{e}=$ $1.5 \cdot 10^{6}$. This implies that the vehicle motion is between the laminar and the turbulent flow [7].

Using laminar theory, we have the following drag coefficient:

$$
C_{D l e}=0.44 \frac{d}{l}+4 C_{f} \frac{l}{d}+4 C_{f}\left(\frac{d}{l}\right)^{\frac{1}{2}},
$$

where $d$ is the vehicle diameter, and $C_{f}$ is given by [9]:

$$
C_{f}=\frac{0.075}{\left(\log _{10} R_{e}-2\right)^{2}}
$$

resulting on $C_{D l e}=0.2$.

The turbulence theory, gives $C_{D t e}=0.08$. The experimental results obtained by [5] to determine the drag coefficient point to a value close to $C_{D l e}$. Taking into account that the dimensions and the speed of operation are similar, we consider:

$$
C_{D}=C_{D l e}
$$

We are now in condition to determine the axial term in $x_{B}$ direction:

$$
\begin{aligned}
& X_{u|u|}=-\frac{1}{2} \rho_{f}\left[2 C_{D e} \int_{-\frac{d}{2}}^{\frac{d}{2}} R_{y z}\left(z_{B}\right) d z_{B}\right. \\
&+2 C_{D e} \int_{\frac{d}{2}}^{y_{m}+\frac{d_{m}}{2}} R_{P y z}\left(y_{B}\right) d y_{B} \\
&+2 C_{D e} \int_{-\frac{d}{2}}^{-y_{m}-\frac{d_{m}}{2}} R_{P y z}\left(y_{B}\right) d y_{B} \\
&\left.+C_{D c S} \int_{z_{S i}}^{z_{S f}} L_{S y z}\left(z_{B}\right) d z_{B}\right]
\end{aligned}
$$

for the limits of integration, see fig.2 and fig.3.

The motion in the $y_{B}$ and $z_{B}$ leads a flow around the vehicle that we approximate by a cylinder. According to [7] and [8], for a cylinder with a ratio $l / d \approx 7.5$, the drag coefficient is:

$$
C_{D C}=0.8
$$


The sonar hull under the vehicle is also cylindrical with a ratio $l_{S} / d_{S} \approx 1$. From [7] and [8], we obtain a drag coefficient equal to:

$$
C_{D c S}=0.68 \text {. }
$$

The drag coefficient terms are determined as shown below:

$$
\begin{gathered}
Y_{v|v|}=-\frac{1}{2} \rho_{f}\left[2 C_{D c} \int_{x_{c}}^{x_{n}} R_{x z}\left(z_{B}\right) d x_{B}+2 C_{D c} \int_{x_{d}}^{x_{C}} R_{P x z}\left(x_{B}\right) d x_{B}\right. \\
\left.+2 C_{D c S} \int_{x_{S i}}^{x_{S f}} H_{S x z}\left(x_{B}\right) d x_{B}\right] \\
M_{q|q|}=-\frac{1}{2} \rho_{f}\left[2 C_{D c} \int_{x_{c}}^{x_{n}}\left|x_{B}\right|^{3} R_{x y}\left(x_{B}\right) d x_{B}\right. \\
\left.+2 C_{D c} \int_{x_{d}}^{x_{m f}}\left|x_{B}\right|^{3} R_{P x y}\left(x_{B}\right) d x_{B}\right] \\
Z_{q|q|}=\frac{1}{2} \rho_{f}\left[2 C_{D c} \int_{x_{c}}^{x_{n}} x_{B}\left|x_{B}\right| R_{x y}\left(x_{B}\right) d x_{B}\right. \\
\left.+2 C_{D c} \int_{x_{d}}^{x_{m f}} x_{B}\left|x_{B}\right| R_{P x y}\left(x_{B}\right) d x_{B}\right] \\
M_{w|w|}=\frac{1}{2} \rho_{f}\left[2 C_{D c} \int_{x_{c}}^{x_{n}} x_{B} R_{x y}\left(x_{B}\right) d x_{B}\right. \\
\left.+2 C_{D c} \int_{x_{d}}^{x_{m f}} x_{B} R_{P x y}\left(x_{B}\right) d x_{B}\right]
\end{gathered}
$$

for the limits of integration, see fig. 2 and fig. 3

The remaining drag terms can be determined by analogy. The most important drag coefficients are shown in table III.

TABLE III

DRAG COEFFICIENT VALUES

\begin{tabular}{|c|c|}
\hline Coefficient & Value \\
\hline$X_{u|u|}$ & $-4.05 \cdot 10^{0}$ \\
\hline$Y_{v|v|}$ & $-1.13 \cdot 10^{2}$ \\
\hline$Z_{w|w|}$ & $-1.15 \cdot 10^{2}$ \\
\hline$K_{p|p|}$ & $-2.29 \cdot 10^{-2}$ \\
\hline$M_{q|q|}$ & $-4.30 \cdot 10^{0}$ \\
\hline
\end{tabular}

\begin{tabular}{|c|c|}
\hline Coefficient & Value \\
\hline$N_{r|r|}$ & $-1.57 \cdot 10^{0}$ \\
\hline$Y_{r|r|}$ & $1.88 \cdot 10^{0}$ \\
\hline$Z_{q|q|}$ & $-5.95 \cdot 10^{0}$ \\
\hline$M_{w|w|}$ & $-8.25 \cdot 10^{0}$ \\
\hline$N_{v|v|}$ & $2.38 \cdot 10^{0}$ \\
\hline
\end{tabular}

\section{LIMITS ANALISYS}

The MARES propellers have a finite actuation force. This fact implies that they reach saturation in some maneuvers. Obviously, these limits depend on vehicle angles, speed and acceleration. In this section, we will study the limits of actuation due to propellers limited force. We will consider particular cases where linear and angular accelerations are null, on maneuver steady-state. The following results will be important whereas we will know the limit relation between the maneuver and speed.

\section{A. Motion at constant depth and pitch}

We consider that the linear and angular acceleration are null, i.e. $\dot{u}=0$. The speeds $u=u_{\text {ref }}$ and $w=w_{\text {ref }}$ are constant. The remaining linear and angular speeds are null.

To maintain a constant depth, it is required that the speed component of $u$ in $z$-axis is compensated by the $w$ component in the same axis. So, we can conclude:

$$
w=u \tan \theta,
$$

where $\theta$ is the pitch angle as we can see in fig.4.

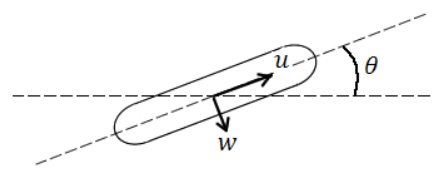

Fig. 4: AUV moving with constant depth and pitch angle

In this analysis, we consider that the body-fixed coordinate system coincides with the center of gravity.

\section{Common mode}

Selecting the third line from (5), we easily get:

$$
f_{p 3}+f_{p 4}=-Z_{|w| w}|u \tan \theta| u \tan \theta+(B-W) \cos \theta .
$$

In steady-state the acceleration in $z_{B}$-axis depends on the drag, propellers and restoring forces. The vertical propellers $p 3$ and $p 4$ are identified in fig. 2 .

\section{Differential mode}

As in common mode determination, we select the fifth line from and we obtain the following expression:

$$
\begin{aligned}
-x_{p 3} f_{p 3}-x_{p 4} f_{p 4}= & -M_{|w| w}|u \tan \theta| u \tan \theta \\
& +\left(Z_{\dot{w}}-X_{\dot{u}}\right) u^{2} \tan \theta-z_{B} B \sin \theta .
\end{aligned}
$$

Assuming that $-x_{p 3} \approx x_{p 4} \approx x_{p}$, we easily get:

$$
\begin{aligned}
& f_{p 3}-f_{p 4} \\
& =\frac{-M_{|w| w \mid}|u \tan \theta| u \tan \theta+\left(Z_{\dot{w}}-X_{\dot{u}}\right) u^{2} \tan \theta-z_{B} B \sin \theta}{x_{p}} .
\end{aligned}
$$

\section{Propeller forces}

Adding and subtracting (26) to (24), we can obtain, respectively:

$$
\begin{aligned}
& f_{p 3} \\
& =\frac{1}{2}\left[-Z_{|w| w}|u \tan \theta| u \tan \theta+(B-W) \cos \theta\right. \\
& \left.+\frac{-M_{|w| w}|u \tan \theta| u \tan \theta+\left(Z_{\dot{w}}-X_{\dot{u}}\right) u^{2} \tan \theta-z_{B} B \sin \theta}{x_{p}}\right], \\
& f_{p 4} \\
& =\frac{1}{2}\left[-Z_{|w| w}|u \tan \theta| u \tan \theta+(B-W) \cos \theta\right. \\
& \left.-\frac{-M_{|w| w}|u \tan \theta| u \tan \theta+\left(Z_{\dot{w}}-X_{\dot{u}}\right) u^{2} \tan \theta-z_{B} B \sin \theta}{x_{p}}\right] .
\end{aligned}
$$

In the next figure we show the propeller force as function of pitch angle $\theta$.

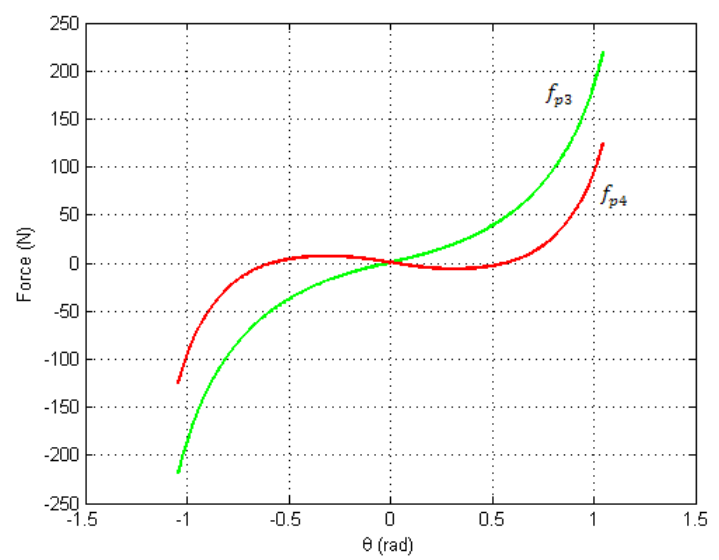

Fig. 5: Force versus pitch angle for a constant speed $u=1 \mathrm{~m} / \mathrm{s}$ 
Assuming that the propellers have a maximum actuation force $\left|f_{\text {pmax }}\right|=30 \mathrm{~N}$, it is possible to determine the maximum angle as a function of $u$. To do this, we solve numerically (27) with $f_{p 3}=\left|f_{p \max }\right|$, taking into account that $p 3$ is the propeller that reaches $\left|f_{\text {pmax }}\right|$ first. The next figure shows the maximum pitch as function of $u$. In this case, we consider only $\theta>0$.

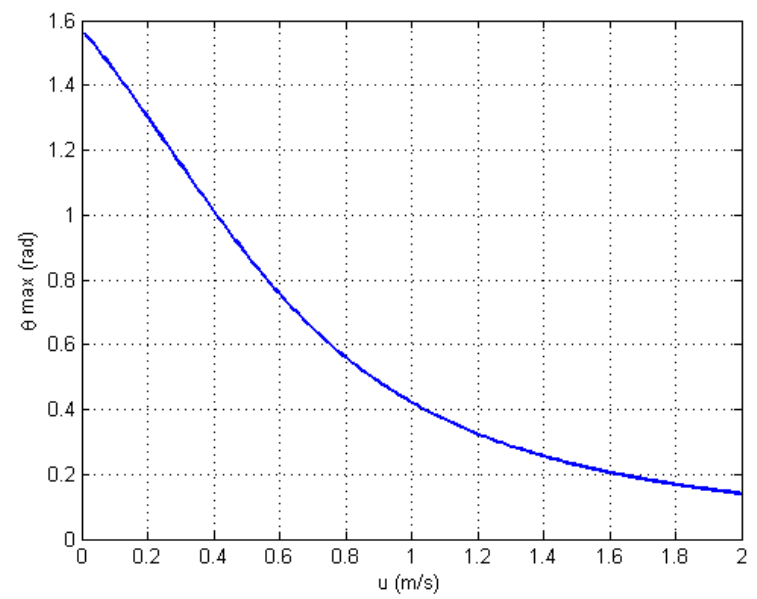

Fig. 6: Maximum pitch angle versus speed

B. Circumference following at constant depth and zero pitch

In this case, we assume that there is no vertical motion and pitch angle is zero, i.e. $z=c_{z}$ and $\theta=0$. The vehicle will have constant surge, sway and yaw speed components in steady-state. Remaining speed components will be zero. Given that, we can conclude that the linear and angular accelerations in body fixed coordinates will be null, in steadystate. So, the yaw speed is given by:

$$
r= \pm \frac{\sqrt{u^{2}+v^{2}}}{R}
$$

where $R$ is the circumference radius described by the vehicle motion. We consider $r>0$. The following results would be symmetric if $r<0$.

Selecting the second line of hydrodynamic equation, we can write:

$$
|v| v=-\frac{\left(X_{\dot{u}} u-m u+Y_{|r| r}|r|\right) r}{Y_{|v| v}}
$$

Substituting $r$ into (30) by (29), we get:

$$
v^{2}= \begin{cases}\frac{\left(X_{\dot{u}} u-m u+Y_{|r| r} \frac{\sqrt{u^{2}+v^{2}}}{R}\right) \frac{\sqrt{u^{2}+v^{2}}}{R}}{Y_{|v| v}}, & v<0 \\ -\frac{\left(X_{\dot{u}} u-m u+Y_{|r| r} \frac{\sqrt{u^{2}+v^{2}}}{R}\right) \cdot \frac{\sqrt{u^{2}+v^{2}}}{R}}{Y_{|v| v}}, & v \geq 0\end{cases}
$$

We will consider only $R$ in the range of 1 to 50 meters. The expression (31) has no solution for $v \geq 0$ for $R \in[1 ; 50]$. Thus, in the range considered:

$$
\operatorname{sign}(v)=-\operatorname{sign}(r)
$$

Defining $\delta$ as the difference between yaw and the circumference tangent angles, as shown in fig.7, we get:

$$
\delta=\operatorname{atan}\left(\frac{v}{u}\right)
$$

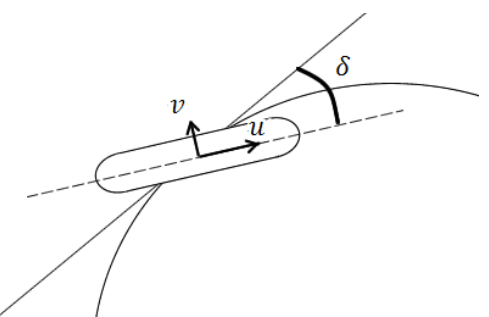

Fig. 7: AUV describing a circumference $(v<0, r>0)$

The fig. 8 shows the evolution of $\delta$ as a function of $R$ for a surge speed $u=1 \mathrm{~m} / \mathrm{s}$.

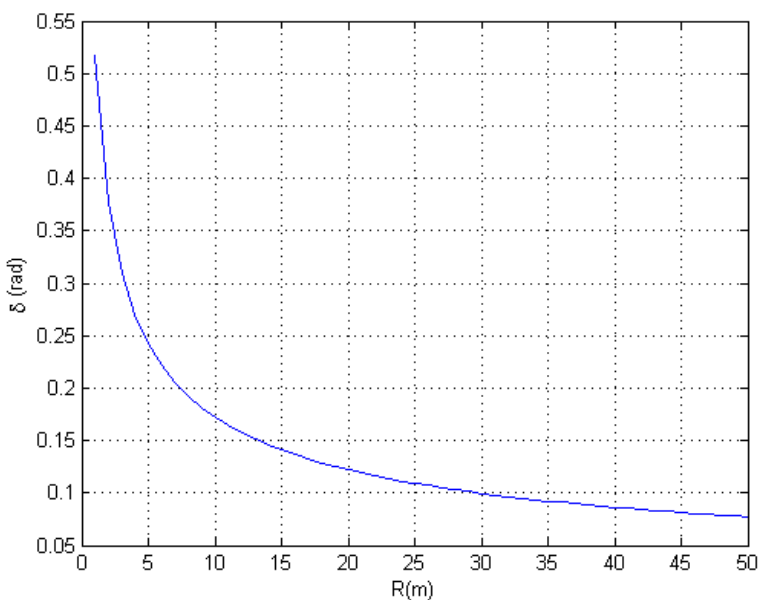

Fig. 8: Difference between yaw and the circumference tangent angles in function of radius

\section{Common mode}

Selecting the first line of hydrodynamic equation (5), we easily obtain:

$$
f_{p 1}+f_{p 2}=\left(Y_{\dot{v}} v+Y_{\dot{r}} r\right) r-m r v-X_{|u| u}|u| u .
$$

The horizontal propellers $p 1$ and $p 2$ are identified in fig. 2 .

\section{Differential mode}

Manipulating the sixth line from (5), the propellers differential mode results:

$$
f_{p 2}-f_{p 1}=\frac{\left(-Y_{\dot{v}} v-Y_{\dot{r}} r\right) u+X_{\dot{u}} u v-N_{|v| v}|v| v-N_{|r| r}|r| r}{y_{p}},
$$

where $y_{p}$ is the distance in $y_{B}$-axis between the propellers center and the center of gravity.

\section{Propeller forces}

By subtracting and adding (35) to (34), we easily obtain, respectively:

$$
\begin{aligned}
& f_{p 1} \\
& =\frac{1}{2}\left[\left(Y_{\dot{v}} v+Y_{\dot{r}} r\right) r-m r v-X_{|u| u}|u| u\right. \\
& \left.-\frac{\left(-Y_{\dot{v}} v-Y_{\dot{r}} r\right) u+X_{\dot{u}} u v-N_{|v| v}|v| v-N_{|r| r}|r| r}{y_{p}}\right],
\end{aligned}
$$




$$
\begin{aligned}
& f_{p 2} \\
& =\frac{1}{2}\left[\left(Y_{\dot{v}} v+Y_{\dot{r}} r\right) r-m r v-X_{|u| u}|u| u\right. \\
& \left.+\frac{\left(-Y_{\dot{v}} v-Y_{\dot{r}} r\right) u+X_{\dot{u}} u v-N_{|v| v}|v| v-N_{|r| r}|r| r}{y_{p}}\right],
\end{aligned}
$$

In the next figure, we show the force applied by each propeller in function of the circumference radius $R$ for $u=1 \mathrm{~m} / \mathrm{s}$.

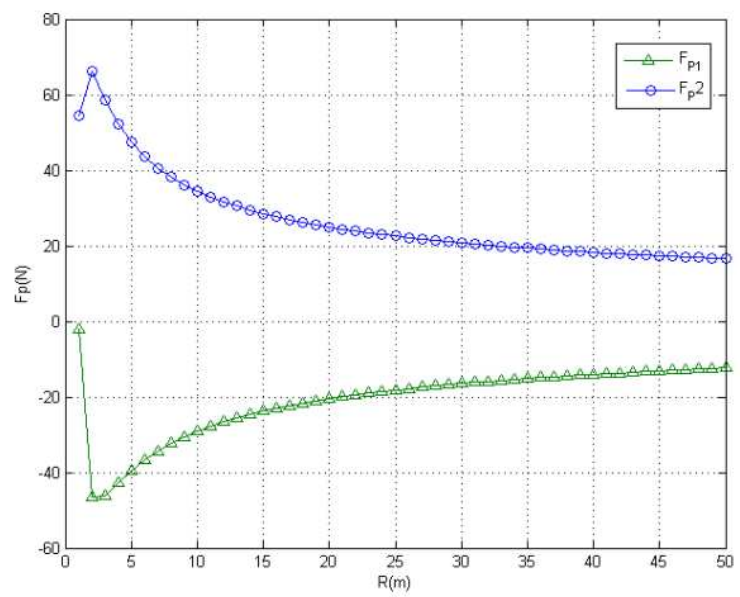

Fig. 9: Applied force for horizontal propellers at $u=1 \mathrm{~m} / \mathrm{s}$

It is not empirical that, for surge and yaw speeds $u, r>0$, the propeller $p 2$ exercise more force than $p 1$ (negative moment after $z_{B}$ ). This result is due to the Munk moment [10$11]$, modeled by terms as $Y_{\dot{v}} v u$ and $X_{\dot{u}} u v$. In practice, this contribution is often compensated by other drag cross-terms that we have neglected in the modeling process, as $N_{u v}$. Authors recommend the reading of [10] and [11] for a more detailed discussion about this subject.

Assuming that the maximum propeller force is $\left|f_{\text {pmax }}\right|$, as in the previous section, we can compute numerically (36) in order to $u$, for $f_{p 1}=\left|f_{p \max }\right|$. We show this result in the next figure as the maximum surge speed as a function of the circumference radius, $R$.

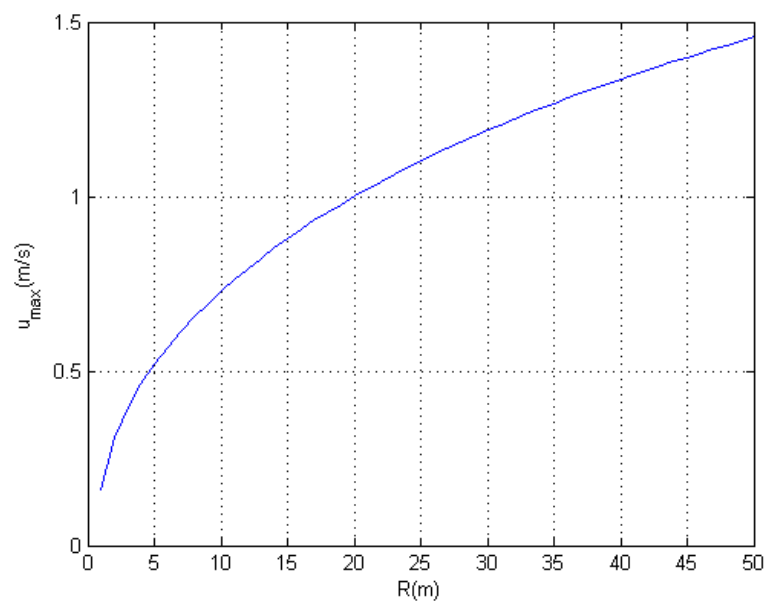

Fig. 10: Maximum surge speed as a function of turning radius

\section{CONCLUSIONS}

We start by presenting AUV MARES, a vehicle designed by the Ocean Systems Group. Investigation in control, oceanographic studies and environmental monitoring areas are the principal aims of this underwater vehicle. We continue with the presentation of the hydrodynamic of a body inserted in a fluid, discriminating the different forces that actuate on it. We follow the commonly used hydrodynamic theory found in [3]. In the coefficients determination section, we compute the added mass and drag coefficients. Though we have relative certainty on the first ones, the others can diverge from experimental results knowing that their theory is based in empirical and experimental formulas for some object forms. The analysis of the motion limits, allows us to approach the behavior of MARES in certain maneuvers. These results are important in order to determine the force requirements and for controllers design.

\section{REFERENCES}

[1] N. Cruz and A. Matos, "The MARES AUV, a Modular Autonomous Robot for Environment Sampling". Québec, Canada : Proceedings of the MTS-IEEE Conference Oceans'2008, 2008.

[2] A. Matos and N. Cruz, "Positioning Control of an Underactuated Surface Vessel". Québec, Canada: Proceedings of the MTS-IEEE Conference Oceans'2008, 2008.

[3] T. I. Fossen, Guidance and control of ocean vehicles. Chichester, England : John Wiley and Sons, 1994.

[4] B. Ferreira, Modelação e controlo de veículo submarino com quatro graus de liberdade. Porto : MSc Thesis, Published by author, 2009.

[5] T. Prestero, Verification of a Six-Degree of Freedom Simulation Model for the REMUS Autonomous Underwater Vehicle. California : s.n., 2001.

[6] D. Yue, Marine Hydrodynamics Lecture 14. Massachussets: MIT Open Courses, 2005.

[7] S. F. Hoerner, Fluid-dynamic drag. USA : edited by author, 1965.

[8] F. M. White, Fluid mechanics. 4th edition. Boston : McGraw-Hill, 1999.

[9] O. M. Faltinsen, Hydrodynamic of high speed marine vehicles. USA : Cambridge, 2005.

[10] M. Triantafyllou and S. Franz, Maneuvering and Control of Marine Vehicles. Cambridge, Massachusetts, USA: edited by authors, 2003.

[11] A.Tyagi, D. Sen, "Calculation of transverse hydrodynamic coefficients using computational fluid dynamic approach". Ocean Engineering, Volume 33, Issues 5-6, April 2006, Pages 798-809.

[12] T. I. Fossen, Nonlinear modelling and control underwater vehicles. Trondheim, Norway : by author, 1991.

[13] B. Allen, W. S. Vorus, e T. Prestero, "Propulsion System Performance Enhancements on REMUS AUVs". Providence R.I.: IEEE Conference Oceans'2000, 2000.

[14] M. Viviani et al. "Identification of hydrodynamic coefficient from standard manoeuvres for a serie of twin-screw ships". Genova, Italy : Unknow, Unknow.

[15] V.A. Underwater vehicles. Austria : edited by A. V. Inzartsev, 2009. 